\title{
Elitist Genetic Algorithm Based Energy Balanced Routing Strategy to Prolong Lifetime of Wireless Sensor Networks
}

\author{
Vinay Kumar Singh ${ }^{1}$ and Vidushi Sharma ${ }^{2}$ \\ ${ }^{1}$ Department of Computer Science \& Engineering and Information Technology, Anand Engineering College, Agra 282007, India \\ ${ }^{2}$ School of Information \& Communication Technology, Gautam Buddha University, Greater Noida 201308, India \\ Correspondence should be addressed to Vinay Kumar Singh; vksingh100@rediffmail.com
}

Received 7 December 2013; Accepted 13 February 2014; Published 16 March 2014

Academic Editors: B.-Y. Cao and I. Smith

Copyright (c) 2014 V. K. Singh and V. Sharma. This is an open access article distributed under the Creative Commons Attribution License, which permits unrestricted use, distribution, and reproduction in any medium, provided the original work is properly cited.

\begin{abstract}
Wireless sensor networks have gained worldwide attention in recent years due to the advances made in wireless communication. Unequal energy dissipation causes the nodes to fail. The factors causing the unequal energy dissipation are, firstly, the distance between the nodes and base station and, secondly, the distance between the nodes themselves. Using traditional methods, it is difficult to obtain the high precision of solution as the problem is NP hard. The routing in wireless networks is a combinatorial optimization problem; hence, genetic algorithms can provide optimized solution to energy efficient shortest path. The proposed algorithm has its inherent advantage that it keeps the elite solutions in the next generation so as to quickly converge towards the global optima also during path selection; it takes into account the energy balance of the network, so that the life time of the network can be prolonged. The results show that the algorithm is efficient for finding the optimal energy constrained route as they can converge faster than other traditional methods used for combinatorial optimization problems.
\end{abstract}

\section{Introduction}

A wireless sensor network (WSN) consists of randomly/ manually deployed sensors that sense the physical or environmental events and send the data to the base station. A large number of small, inexpensive, disposable, and autonomous sensor nodes are generally deployed in an ad hoc manner in vast geographical areas for remote operations. Sensor nodes in a WSN are constrained in storage capacity, computation power, bandwidth, and power supply [1-3]. The development of low-cost, low-power, multifunctional sensor has received increasing attention from various industries. Sensor nodes are smaller in size and capable of sensing, gathering, and processing data. They also communicate with other nodes in the network, via radio frequency (RF) channel. The areas of applications of WSNs vary from civil, healthcare, and environmental to military [4].

Recent advances in WSN have led to searching for new routing schemes for wireless sensors where energy awareness is essential consideration. Traditional networks aim to achieve high quality of service (QoS) provisions; thus sensor network schemes must focus primarily on power conservation. Though there are some similarities between the WSN and ad hoc network, like both are multihop communications, they differ in many ways. Some of the power-aware routing protocols proposed for ad hoc networks can be examined for energy constraints, but ad hoc routing techniques proposed in the literature are not effective, feasible and do not usually fit the requirements of the sensor networks. The applications and routing requirements for the two are different in various aspects; first, the typical mode of communication in a sensor network is from multiple data sources to a data recipient/sink rather than communication between any pair of nodes. Second, since the data being collected by multiple sensors is based on common phenomena, there is likely to be some redundancy in the data being communicated by the various sources in sensor networks. Third, in most envisioned scenarios the sensors are not mobile, so the nature of the dynamics in the two networks is different. Finally, the single major resource constraint in sensor networks is that of energy. The situation is much worse than in traditional wireless networks, where the communicating devices handled by human users 
can be replaced or recharged relatively often. The scale of sensor networks and the necessity of unattended operation for months at a time mean that energy resources have to be managed even more carefully. This, in turn, precludes high data rate communication and demands energy-efficient routing protocols. Hence, it is necessary to design a special multihop wireless routing technique between the sensor nodes and the sink node with a focus on energy efficiency.

A typical energy-efficient routing protocol can be described in the following three phases as shown in Figure 1. In the first phase, sink broadcasts the interest or sensor nodes broadcast an advertisement for the available data and wait for a response. In the second phase, if the observations of some sensor nodes are matched with the interest list propagated by the sink, then the nodes forward data packets containing the required information to the sink using a certain routing scheme. In the third phase, the sink infrequently initiates a localized flooding in the network in order to keep all paths alive. The second phase is primarily considered due to the fact that data forwarding consumes much more energy than the two other phases.

\section{Literature Review}

Energy-efficient routing in wireless sensor networks has gained a lot of attraction from the researchers in the recent years. A summary of recent research results on energy efficient data routing in sensor networks is discussed in [5, 6]. To increase the network lifetime, the design of efficient routing protocol for communication is very important. An illustration of the existing routing protocol for wireless sensor network using data centric approach and present performance analysis of these protocols is done in [7], which may have been further improved by considering optimization of other parameters like cost, distance, and so forth along with energy optimization. Evolutionary Algorithms can be used effectively to find the energy efficient path in wireless sensor networks [8]. A simple approach to minimize the average path length is proposed in [9] where they considered the wireless network of transceiver nodes with a known spatial distribution using a genetic algorithm optimization method. Each of the nodes consists of a relatively simple a transceiver (antennas, a receiver, and a transmitter). The goal of the optimization is to minimize the average path length from source to destination to minimize the transmitted power. This may sometimes result in early depletion of energy in some nodes in the shortest path selected for data communication. Further, a method proposed in [10] has used a multipath routing protocol for wireless sensor networks that enhances the reliability of WSN by using multipath routing. It is useful for delivering data in unreliable environments. The idea is to define many paths from source to sink and send through them the same subpackets. This implies that the traffic will increase significantly, at the same time it will increase the reliability of the network. The idea is to split the original data packet into subpackets through each path. This can offer at the end, even with the loss of subpackets, the reconstruction of the original message. The only drawback of this strategy is that it is not energy aware and provides more reliability at the cost of more energy depletion. Energy aware consideration is not done which may reduce the lifetime of the sensor network. The energy awareness in multipath routing is done in [11-14] with consideration of maximum lifetime routing in wireless sensor networks. This protocol routes data through a path whose nodes have the largest residual energy. The path is switched whenever a better path is discovered. The primary path will be used until its energy is below the energy of the backup path. By means of this approach, the nodes in the primary route will not exhaust their energy by the continual use of the same path, thus achieving longer lifetime.

Recent advances in WSN have led to searching for new routing schemes for wireless sensors where energy awareness is essential consideration [15]. The aim of networks is to achieve high quality of service (QoS) communication; thus, sensor network schemes must focus primarily on power conservation. Ad hoc routing techniques proposed in the literature do not usually fit the requirements of the sensor networks. Hence, it is necessary to design a special multihop wireless routing technique between the sensor nodes and the sink node with a focus on energy efficiency. A clustering method is proposed in [16] where a fixed number of cluster heads are selected based on maximum residual energy of the nodes. Each cluster head is associated with a group of nodes based on the minimum distance among them. In such scheduling, all the nodes dissipate uniform energy and subsequently remain alive for long time. The energy load balancing strategy used in this paper not only ensures the balanced energy consumption of the nodes in the network so that the network lifetime is prolonged, but it also considers the minimum cost path towards the sink so that energy can be further saved and elitism-based GA ensures faster convergence.

The rest of the paper is organized as follows. Section 3 describes the network model used in this paper, Section 4 talks about the modified GA approach with elitism used for WSN routing, Section 5 provides and analysis of the results obtained using the proposed scheme, and Section 6 is the conclusion.

\section{The Proposed Network Model}

The model considered in this paper realizes a powerful Base Station which has adequate source of energy supply and it is located far away from the sink. The sensor nodes are homogeneous and have limited energy. All the nodes have uniform initial energy allocation. The first-order radio model is used for calculation of the energy dissipation for data communication operations like transmission and reception. The energy spent by a node in transmitting a $k$-bit packet to another node $d$ meters away is given by

$$
\operatorname{ETX}(k, d)=\left(\xi_{\text {elec }}+\xi_{\text {amp }} * d_{i}^{n}\right) * k
$$

and the energy spent in receiving the packet is

$$
\operatorname{ETX}(k)=\xi_{\text {elec }} * k
$$




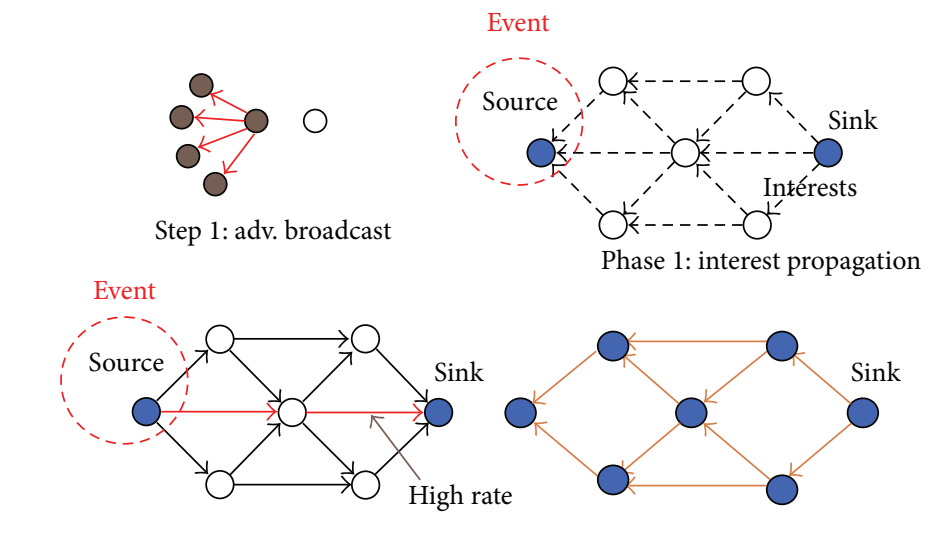

Phase 2: data delivery along reinforced path Phase 3: localized directional flooding

FIGURE 1: Energy-efficient routing.

The energy spent in transmitting a packet from node $i$ to node $j$ is the same as that from node $j$ to node $i$ for any given value of SNR; that is, there is a symmetric channel. The computational energy is negligible in comparison to communicational energy and can be ignored. A routing path or chain is an ordered sequence of the nodes in the network that transmit the data message towards the sink node. A time interval in which a node generates a packet and transmits it towards its neighbour is called a data collecting round. After receiving a packet from its previous node, every node relays it to the next node in the chain. The task is to minimize the energy dissipation in the nodes, by the formation of an optimal data collecting chain. Considering $N$ nodes in the network, the total energy expended in a typical data gathering round is the summation of the energy dissipated by the individual sensor nodes. Total energy spent can be calculated for a packet size of $k$ as follows:

$$
E_{\text {TOTAL }}=\left\{\sum_{i=1}^{N-1}\left(\xi_{\text {elec }}+\xi_{\text {amp }} * d_{i}^{n}\right)+\xi_{\text {elec }}\right\} * k,
$$

where $d_{i}$ denotes the distance between the $i$ th node and the $(i+1)$ th node in the data collecting chain. A threshold value is imposed on the communication radius between the nodes as $d$ th. This is determined by the communicating radius limit of the sensors as the sensor nodes are not able to transmit or receive reliably beyond this limit. This ensures reliable communication in between the nodes reducing unwanted noise and packet loss probability also this is necessary to reduce the energy consumption.

\section{Improved Genetic Algorithm}

Genetic algorithms (GAs) are stochastic search mechanisms. They are mainly a probabilistic search and optimization technique inspired by the mechanics of (Darwinian) natural selection and genetics [17]. It has been shown [18-20] that genetic algorithm performs well in solving the problem of finding path optimization for WSN. It works on a group of optimal or suboptimal solutions during the search and gives the optimal search path within a short time.
The algorithm works by storing useful information about the individuals from the current population either implicitly through redundant representations [21-23]. The elitist strategy ensures that the best individual will not be destroyed. Instead, the best individual will take place of the worst individual after the operations of crossover and mutation. If the energy of a particular node in the routing reaches below a predefined level, the node is replaced in the routing chain based on some probability. This is done, to prolong the lifetime of the network. Also new individuals are inserted into the population after every generation so that the diversity is maintained and GA does not get converge into local optima as shown in Figure 2. The steps of the modified GA are as follows.

(i) Population Initialization. In the first step $N$ individuals (chromosomes) are produced randomly and the evolutionary generation starts with iteration 0 . The distance threshold (communication radius) is initialized.

(ii) Fitness Calculation. The fitness function is designed to increase the lifetime of the network, which evaluates whether a particular chromosome increases lifetime of the network or not. The algorithm preserves the historically obtained best chromosome; that is, with the highest fitness value this is called elitism. The fitness of each chromosome is calculated by

$$
C_{f}=\sum_{i=1}^{N-1} d_{i}^{2}
$$

The above equation calculates the energy of a chromosome $C$ containing $N$ genes and $d_{i}$ denotes the distance between the $(i+1)$ th node (or, gene) and the $i$ th node in the data gathering chain. A greater value of the chromosome energy indicates a longer data gathering chain which means an inferior solution.

The energy balance for each individual is computed by the sum of the residual energy $P_{i}$ of all the nodes in the individual divided by the total number of nodes:

$$
E_{f}=\frac{\sum_{i=1}^{N} P}{N} .
$$




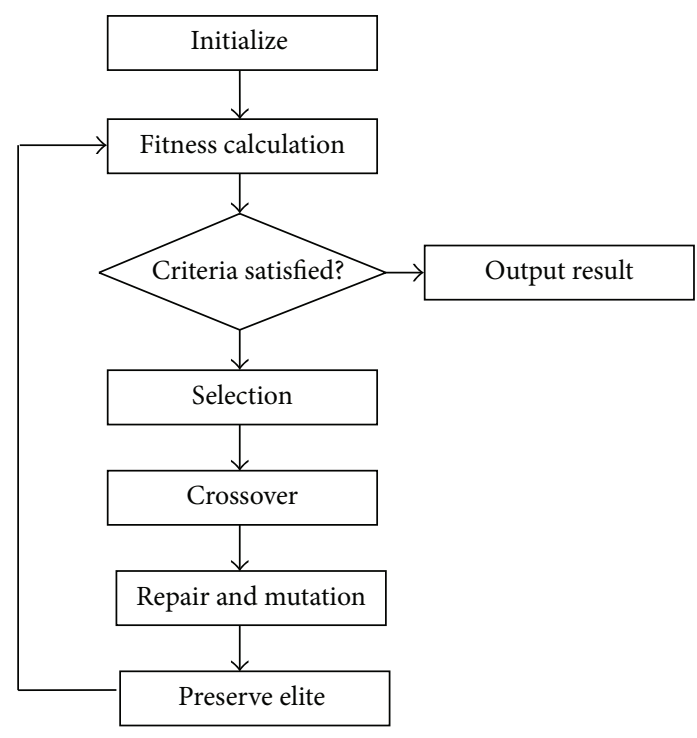

Figure 2: Flowchart of modified genetic algorithm.

The fitness function which takes into account the cost (proportional to the distance between the nodes) between the nodes as well as the energy balance is thus given by

$$
F(C, E)=C_{f}+E_{f}
$$

(iii) Selection. This step selects the better individuals according to the corresponding selection operator. The selection operator used here is tournament selection where by a mating pool of the individuals with above average fitness value is maintained and two parents are randomly selected for crossover.

(iv) Crossover. This step produces the new individuals to complete the global search according to the corresponding crossover operator. Two-point crossover operator is used in this paper. In two-point crossover two points are randomly selected in a chromosome and all the genes from one point to another point are copied from the first chromosome to the second chromosome.

(v) Repair. This step removes the routing loop. The genes values which are repeated in the chromosome are deleted and thus the looping is removed. If a child violates the imposed constraints (distance threshold), then it is rejected and again the crossover is performed.

(vi) Mutation. This step produces the new individuals to maintain the diversity in the population. In mutation, a node is randomly selected from the best chromosome obtained in the past generation and a gene value is changed randomly to produce a new offspring. If the new offspring violates the constraints like distance threshold, it is rejected and mutation is performed again using repair function. The mutation operation can help the search to exit out of the local optima and search for global optima.

(vii) Elitism. In this step the best solutions from the population are maintained in the next generation so that the convergence is faster.

(viii) Checking the Terminating Criteria. If the maximum number of generations has elapsed or the energy of the network has fallen below predefined level, the algorithm is terminated.

(ix) Producing the Result. The program outputs the best chromosome found so far as the result.

\section{Simulation Results}

The energy dissipated per bit (in (3)) to run the radio electronics is $\xi_{\text {elec }}(50 \mathrm{~nJ} / \mathrm{bit})$ and $\xi_{\text {amp }}\left(10 \mathrm{pJ} / \mathrm{bit} / \mathrm{m}^{2}\right)$ is the energy required by the transmit amplifier to maintain an acceptable signal to noise ratio (SNR) in order to transfer data messages reliably. $n$ is called the path loss exponent, whose value enhances with increasing channel nonlinearity (usually, $2.0 \leq n \leq 4.0$ ). The value of $n$ is 2 for free space transmission. The simulations are done under the MATLAB programming environment. Custom code is written to simulate the wireless sensor network. The number of sensor nodes varies from 10 to 50 . The sensors are randomly distributed in the area of $100 \mathrm{~m} \times 100 \mathrm{~m}$. The sink node is at $(100,100)$. The initial energy of sensor nodes is $1 \mathrm{~J}$. Every node transmits ten 4000 bits messages every round. The number of individuals in the population is the same as that of total number of nodes in the network, and the maximum number of generations for genetic algorithm is ten times as that of total number of nodes in the network.

The paper shows the comparison of different energyefficient routing schemes for WSN: (a) direct transmission between sensor nodes and the sink, (b) genetic algorithm based routing with least average energy consumption (ELGA) [17], (c) genetic algorithm based routing with energy balance being taken into account (EBGA) [17], and (d) Elitism based GA routing which takes into account the cost (distance) between the nodes as well as the energy balance of the individual (routing). Figure 3 shows the comparison of the following four situations mentioned above. The algorithms are tested for up to maximum of 50 nodes. It is observed that there is much improvement in the network lifetime using the proposed GA with elitism. There is 6\% improvement in the network lifetime with the proposed scheme for 50 nodes, as compared to EBGA, whereas with direct method it is $208 \%$ improvement which is more significant. Figure 4 shows the residual energy of twenty nodes for $100 \mathrm{~m} \times$ $100 \mathrm{~m}$ network area when the first dead node appears. The average residual energy of the network using direct, ELGA, and EBGA method is $0.6972 \mathrm{~J}, 0.3791 \mathrm{~J}$, and $0.0184 \mathrm{~J}$ [17]. The average energy of the network using the proposed algorithm is $0.1530 \mathrm{~J}$ which is $16.8478 \%$ improvement over the EBGA. This shows that the proposed algorithm better utilizes the network energy which increases the network 


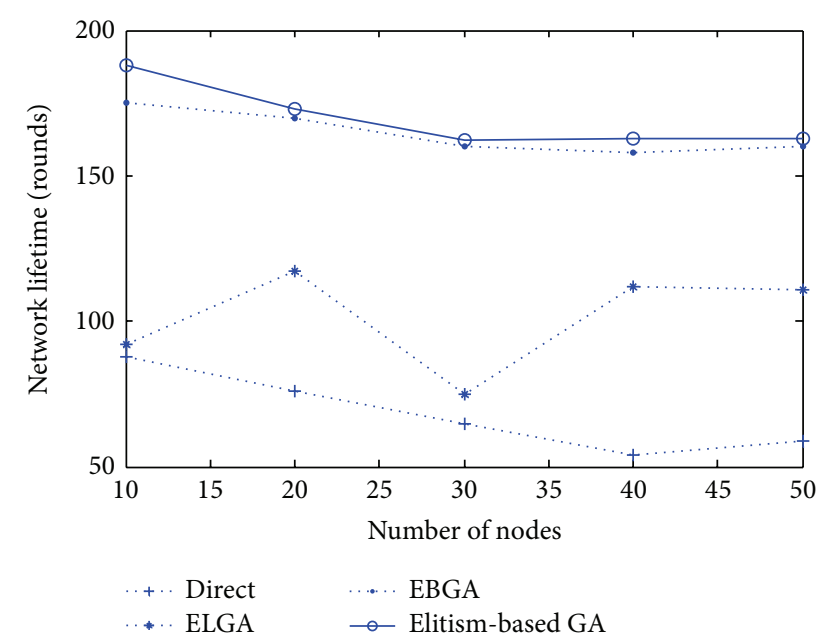

FIGURE 3: Network lifetime versus number of nodes; comparison of different routing schemes.

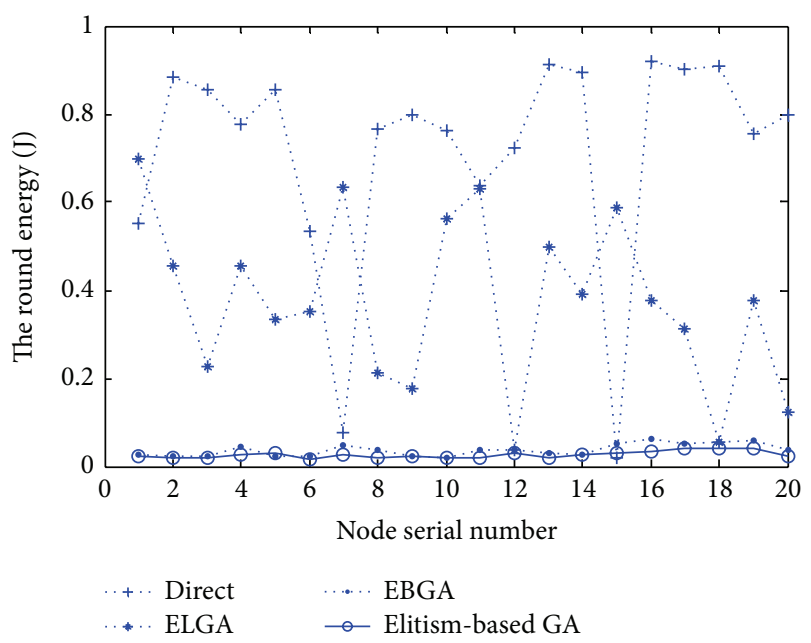

Figure 4: Comparison of residual energy of nodes under different routing schemes.

lifetime significantly. When finding the optimized routing, the elitism-based GA using the fitness criteria mentioned in (6) converges much faster as compared to simple GA for the same fitness criteria. This is because it preserves the best solution for the next generation which improves the GA to a greater extent as shown in Figure 5. The simple GA converges in about 70 generations whereas the elitism-based GA takes about 20 generations to converge, which is much faster.

\section{Conclusion}

In this paper, the basic genetic algorithm is improved using the elitism concept to provide the solution to combinatorial optimization problem of finding the energy efficient shortest routing for the wireless sensor networks. The modified GA uses elitist strategy so that the best individual is preserved and carried to the next generation so that there is significant

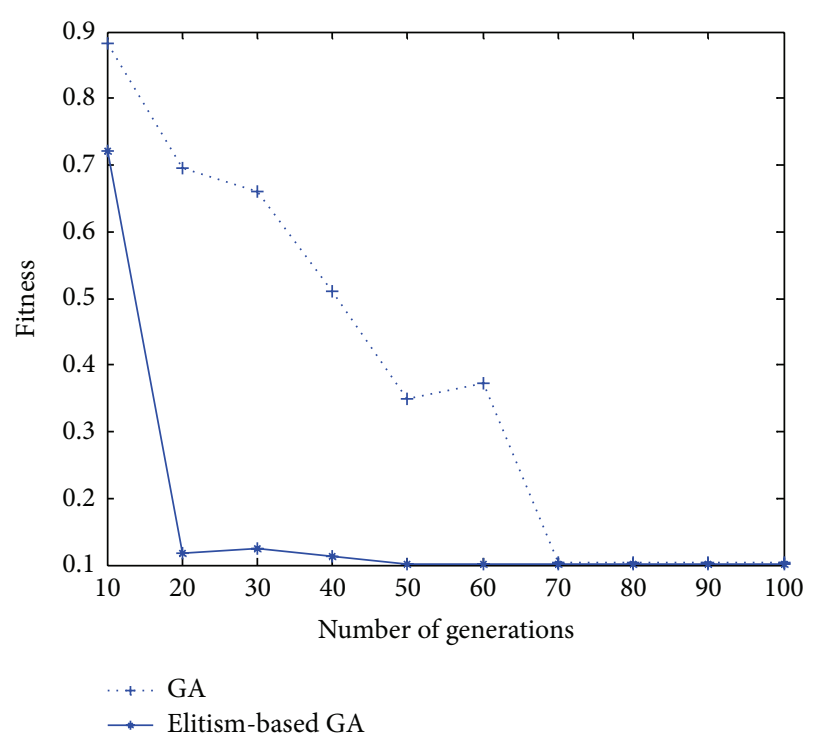

FIGURE 5: Comparison of convergence of simple GA and elitismbased GA.

improvement in the convergence. The node energy falling below predefined level is restrained from the path based on some probability to increase the overall lifetime of the network. The energy load balancing strategy prevents uneven energy dissipation and thus the network lifetime is prolonged considerably.

\section{Conflict of Interests}

The authors declare that there is no conflict of interests regarding the publication of this paper.

\section{References}

[1] D. E. Goldberg, Genetic Algorithms in Search, Optimization, and Machine Learning, Addison-Wesley, Reading, Mass, USA, 1989.

[2] I. F. Akyildiz, W. Su, Y. Sankarasubramaniam, and E. Cayirci, "A survey on sensor networks," IEEE Communications Magazine, vol. 40, no. 8, pp. 102-105, 2002.

[3] R. V. Kulkarni, A. Förster, and G. K. Venayagamoorthy, "Computational intelligence in wireless sensor networks: a survey," IEEE Communications Surveys and Tutorials, vol. 13, no. 1, pp. 68-96, 2011.

[4] S. R. Heikalabad, A. Ghaffari, A. Hadian, and H. Rasouli, "DPCC: dynamic predictive congestion control in wireless sensor networks," International Journal of Computer Science Issues, vol. 8, no. 1, pp. 472-477, 2011.

[5] R. Min, M. Bhardwaj, S.-H. Cho et al., "Low-power wireless sensor networks," in Proceedings of the 14th International Conference on VLSI Design, pp. 221-226, Bangalore, India, January 2001.

[6] K. Akkaya and M. Younis, "A survey on routing protocols for wireless sensor networks," Elsevier Ad Hoc Networks, vol. 3, no. 3, pp. 325-349, 2005.

[7] A. H. Azni, M. M. Saudi, A. Azman, and A. S. Johari, "Performance analysis of routing protocol for WSN using data 
centric approach," World Academy of Science, Engineering and Technology, no. 53, 2009.

[8] V. K. Singh and V. Sharma, "On the hybridization of evolutionary algorithms and optimization techniques," in Proceedings of the COMMUNE Conference on Advancements in Communication, Computing \& Signal Processing (CACCS '11), April 2011.

[9] J. M. Johnson and Y. Rahmat-Samii, "Genetic algorithm optimization of wireless communication networks," in Proceedings of the 1995 IEEE Antennas and Propagation Society International Symposium, AP-S. Digest, pp. 1964-1967, June 1995.

[10] Q. Li, J. Aslam, and D. Rus, "Online power-aware routing in wireless ad-hoc networks," in Proceedings of the DIMACS Workshop on Pervasive Networking, 2001.

[11] S. Murthy and J. J. Garcia-Luna-Aceves, "An efficient routing protocol for wireless networks," Mobile Networks and Applications, vol. 1, no. 2, pp. 183-197, 1996.

[12] S. Singh, M. Woo, and C. S. Mghavendra, "Power-aware routing in mobile ad hoc networks," in Proceedings of the 4th Annual ACM/IEEE International Conference on Mobile Computing and Networking (MobiCom '98), pp. 181-190, ACM SIGMOBILE Mobile Computing and Communications Review, 1998.

[13] W. R. Heinzelman, A. Chandrakasan, and H. Balakrishnan, "Energy-efficient communication protocol for wireless microsensor networks," in Proceedings of the 33rd Annual Hawaii International Conference on System Siences (HICSS '00), pp. 876-882, IEEE, January 2000.

[14] J. Chang and L. Tassiulas, "Maximum lifetime routing in wireless sensor networks," IEEE/ACM Transactions on Networking, vol. 12, no. 4, pp. 609-619, 2004.

[15] J. Yick, B. Mukherjee, and D. Ghosal, "Wireless sensor network survey," Computer Networks, vol. 52, no. 12, pp. 2292-2330, 2008.

[16] P. Azad, B. Singh, and V. Sharma, "A novel clustering approach for extending the lifetime for wireless sensor networks," International Journal of Advances in Engineering and Technology, vol. 1, no. 5, pp. 441-446, 2011.

[17] W. Guo, H. Shi, J. Yan, and Y. Zhou, "Application of genetic algorithm in energy-efficient routing," in Proceedings of the China-Japan Joint Microwave Conference (CJMW'08), pp. 737740, September 2008.

[18] L. Guo and Q. Tang, "An improved routing protocol in WSN with hybrid genetic algorithm," in Proceedings of the 2nd International Conference on Networks Security, Wireless Communications and Trusted Computing (NSWCTC '10), vol. 2, pp. 289-292, April 2010.

[19] O. Islam and S. Hussain, "An intelligent multi-hop routing for wireless sensor networks," in Proceedings of the IEEE/WIC/ACM International Conference on Web Intelligence and Intelligent Agent Technology Workshops (WI-IAT '06), pp. 239-242, December 2006.

[20] M. Al-Ghazal, A. El-Sayed, and H. Kelash, "Routing optimization using genetic algorithm in ad hoc networks," in Proceedings of the IEEE Symposium on Signal Processing and Information Technology (ISSPIT '07), pp. 497-503, Cairo, Egypt, December 2007.

[21] D. Dasgupta and D. McGregor, "Nonstationary function optimization using the structured genetic algorithm," in Proceedings of the 2nd International Conference on Parallel Problem Solving Nature, pp. 145-154, 1992.

[22] E. Lewis and G. Ritchie, "A comparison of dominance mechanisms and simple mutation on non-stationary problems," in Proceedings of the 5th International Conference on Parallel Problem Solving Nature, pp. 139-148, 1998.
[23] A. Uyar and A. E. Harmanci, "A new population based adaptive domination change mechanism for diploid genetic algorithms in dynamic environments," Soft Computing, vol. 9, no. 11, pp. 803-815, 2005. 

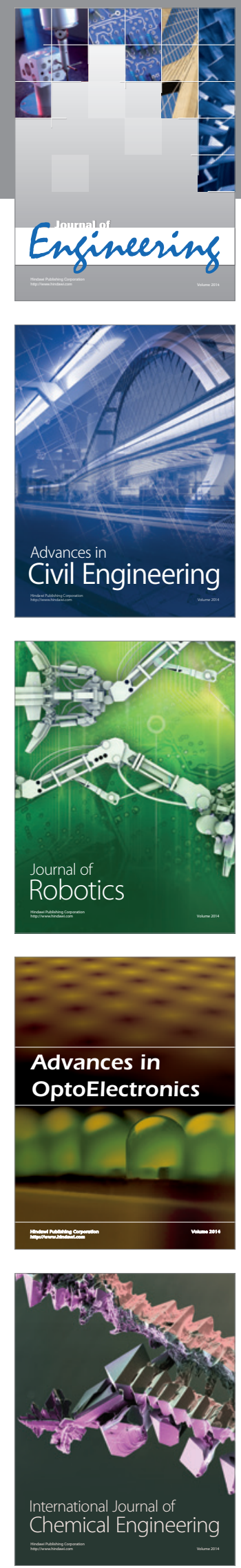

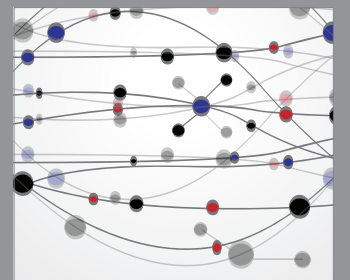

The Scientific World Journal
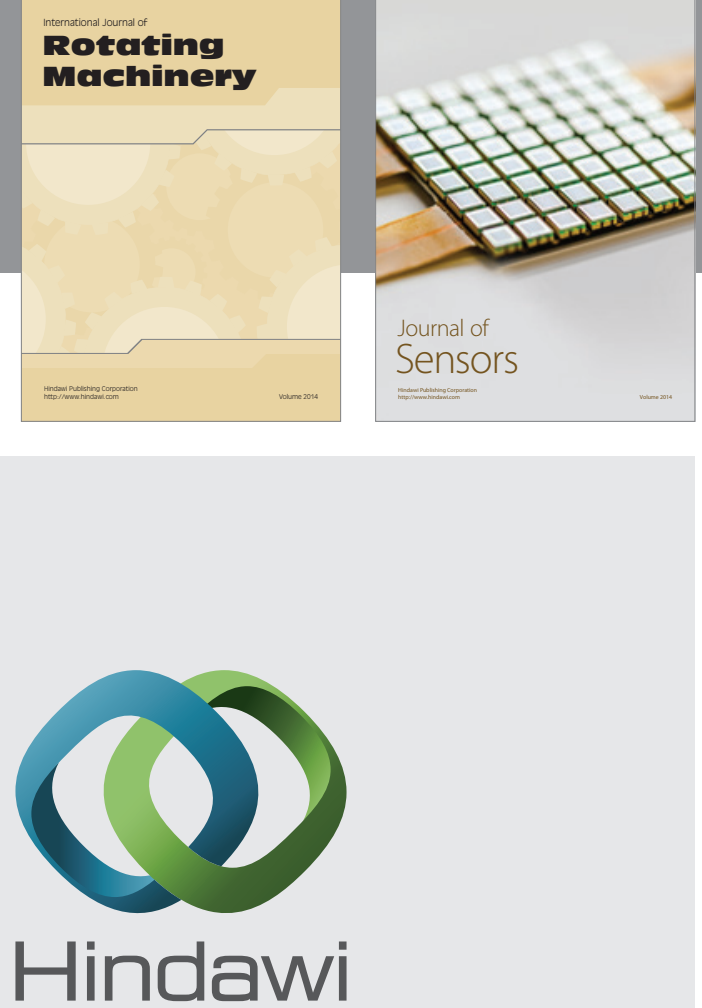

Submit your manuscripts at http://www.hindawi.com
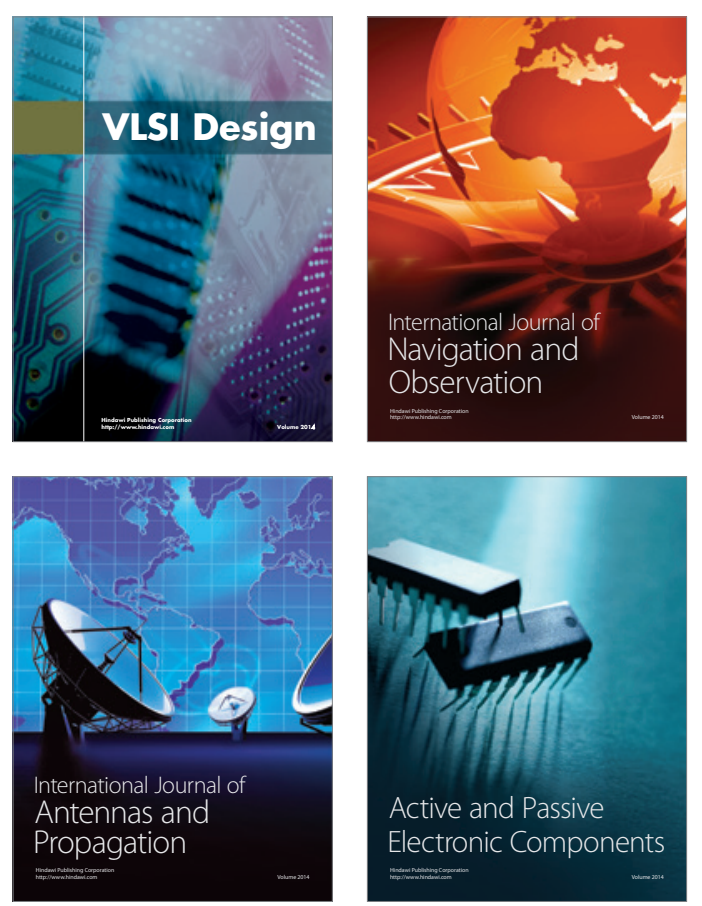
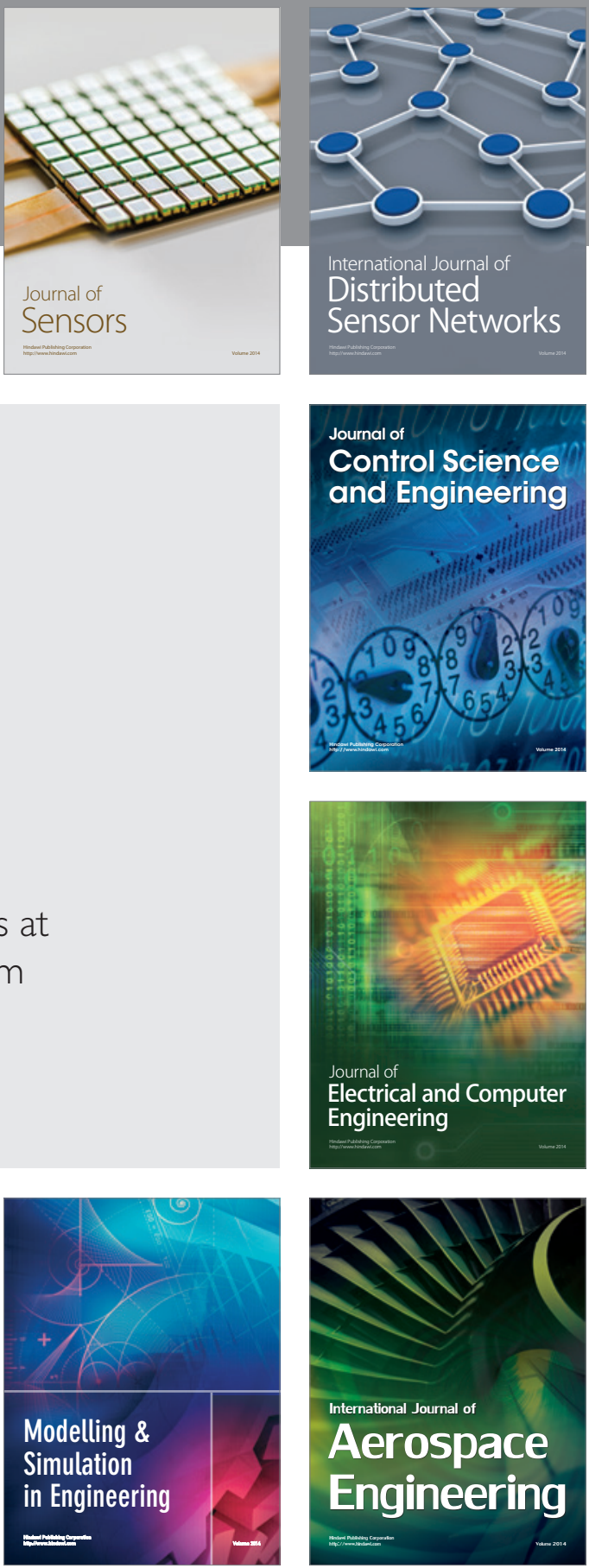

Journal of

Control Science

and Engineering
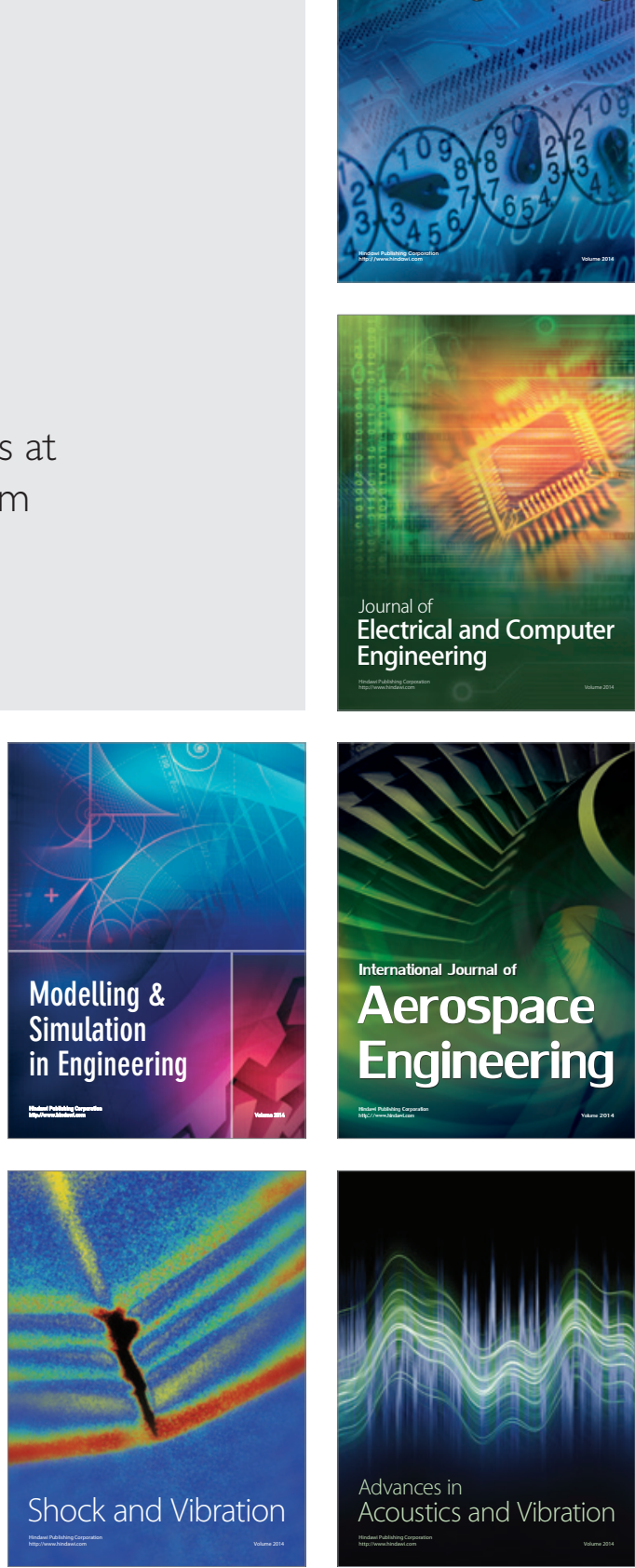